\title{
NOUVELL
}

\section{Rôle de l'autophagie des cellules endothéliales sinusoïdales du foie dans la stéatohépatite non alcoolique}

\author{
Adel Hammoutene ${ }^{1,2}$, Pierre-Emmanuel Rautou ${ }^{1-4}$
}

> La stéatose hépatique non alcoolique (nonalcoholic fatty liver disease, NAFLD) est la manifestation hépatique du syndrome métabolique. La forte prévalence de la NAFLD dans la population générale, d'environ $25 \%$, constitue un problème de santé publique [1]. La NAFLD est associée à un large spectre de lésions histologiques allant d'une simple accumulation de lipides (stéatose) sans lésion hépatocytaire, inflammation ou fibrose, à la stéatohépatite non alcoolique (nonalcoholic steatohepatitis, NASH), qui comporte, en plus de la stéatose, une atteinte hépatocytaire et une inflammation avec des degrés variables de fibrose hépatique $[2,3]$. La prévalence de la NASH dans la population générale est estimée à $5 \%$, et est en constante augmentation à l'échelle mondiale. L'évolution de la NASH se ferait dans $25 \%$ des cas vers la cirrhose et le carcinome hépatocellulaire, pouvant nécessiter une transplantation hépatique [1-3]. La physiopathologie de la NASH reste mal comprise, en particulier les mécanismes responsables de la transition de la stéatose pure vers la NASH.

Les cellules endothéliales sinusoïdales $\mathrm{du}$ foie (liver sinusoidal endothelial cells, LSEC) sont des cellules spécialisées qui séparent les hépatocytes et les cellules étoilées hépatiques du sang en provenance de l'intestin et du tissu adipeux viscéral $[4,5]$. En conditions physiologiques, les LSEC contribuent à l'homéostasie du foie par leurs propriétés anti-inflammatoires et antifibrogéniques. Le syndrome métabolique altère les $L S E C$ par des mécanismes qui ne sont pas encore totalement élucidés. Ces cellules acquièrent alors de nouvelles propriétés pro-inflammatoires et pro-fibrogéniques contribuant au développement de la NASH [4]. Dans le but de mieux comprendre le rôle des LSEC dans la NASH, nous avons analysé, par microscopie électronique, des biopsies de foie de patients atteints ou non de cette maladie, et avons alors noté la présence de vacuoles d'autophagie dans le cytoplasme des LSEC. L'autophagie est un processus physiologique de dégradation, par lequel la cellule recycle en permanence son matériel cytoplasmique dysfonctionnel, tout en évitant l'accumulation d'agrégats protéiques ou d'organelles anormales. Ce processus est rapidement activé en cas de carence en nutriments ou de stress cellulaire. Dans les hépatocytes et les macrophages résidents du foie (cellules de Kupffer), l'autophagie protège contre la NASH en évitant l'accumulation de lipides dans les hépatocytes et en empêchant l'activation des cellules de Kupffer. Dans les cellules étoilées du foie, l'autophagie est pro-fibrogénique en contribuant à leur activation [6]. Bien que les LSEC soient parmi les premières cellules hépatiques altérées dans la NASH, la contribution à la maladie de l'autophagie dans ces cellules était inconnue. Nous avons donc quantifié le nombre de vacuoles d'autophagie dans les LSEC de patients atteints de NASH, et avons observé environ deux fois moins de LSEC contenant des vacuoles d'autophagie chez ces patients que chez
${ }^{1}$ Université de Paris, PARCC, Inserm,

75015 Paris, France.

${ }^{2}$ Université de Paris, Centre de recherche sur I'inflammation, Inserm U1149, CNRS ERL8252, 75018 Paris, France.

${ }^{3}$ Service d'hépatologie, département médicouniversitaire Digest, Hôpital Beaujon

(AP-HP), 100 boulevard du Général Leclerc, 92100 Clichy, France.

${ }^{4}$ Centre de référence des maladies vasculaires du foie, réseau français de référence pour les maladies rares du foie (FILFOIE), réseau européen de référence pour les maladies hépatiques (ERN RARE-LIVER), Paris, France. adel.hammoutene@inserm.fr

pierre-emmanuel.rautou@inserm.fr

des individus dont le foie est sain, ou que chez les patients atteints d'une stéatose pure [7]. Une analyse morphométrique a par ailleurs montré que les vacuoles d'autophagie présentes dans les LSEC de patients atteints de NASH étaient de taille réduite par rapport à celles des LSEC d'individus témoins et de patients atteints de stéatose pure, ce qui indique que le flux autophagique des LSEC est diminué en cas de NASH [7]. Cette observation, qui suggère l'existence d'un défaut d'autophagie dans les LSEC des patients atteints de NASH, nous a conduits à formuler l'hypothèse que ce défaut pourrait contribuer aux altérations endothéliales qui précèdent la maladie, et favoriser l'évolution de la stéatose hépatique vers la NASH.

Nous avons cherché à identifier les facteurs responsables de ce défaut d'autophagie dans les LSEC, et avons montré que la combinaison des cytokines inflammatoires TNF- $\alpha$ (tumor necrosis factor $\alpha$ ) et interleukine 6 (IL-6), qui sont impliquées dans la physiopathologie de la NASH, diminuait de $50 \%$ le niveau d'autophagie dans une lignée de LSEC en culture [7]. Nous avons ensuite analysé les conséquences du défaut d'autophagie sur le phénotype des LSEC en utilisant deux approches complémentaires. Nous avons d'abord montré, par des expériences in vitro, que des LSEC génétiquement déficientes pour l'autophagie surexprimaient des gènes codant des protéines de l'inflammation ( $C \mathrm{CL}[\mathrm{C}$ - 


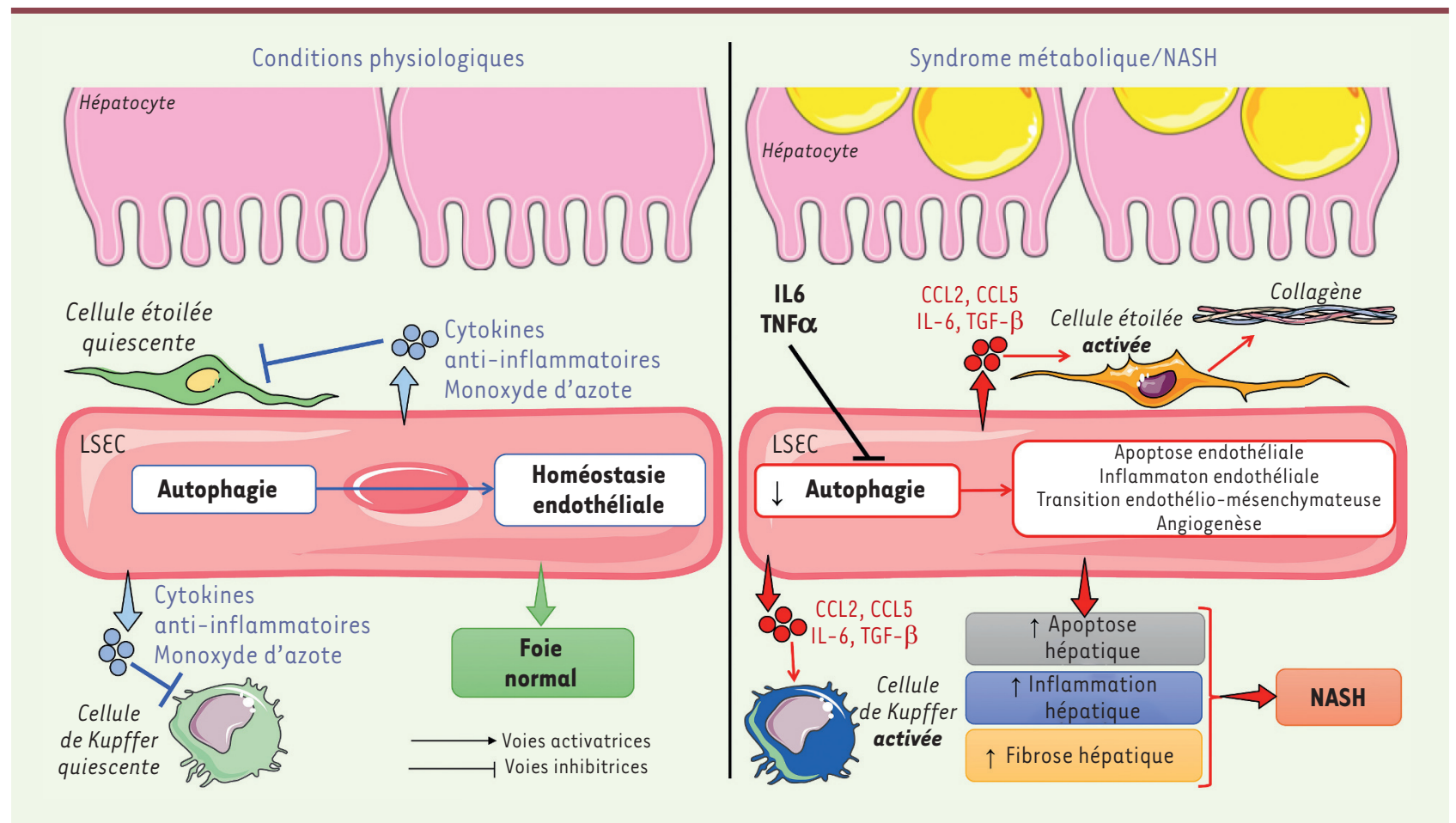

Figure 1. Rôle de l'autophagie des cellules endothéliales sinusoïdales du foie (LSEC) dans la NASH. En conditions physiologiques, l'autophagie dans les LSEC contribue au maintien de l'homéostasie endothéliale et hépatique. Les LSEC maintiennent la quiescence des cellules de Kupffer et des cellules étoilées du foie en libérant des molécules anti-inflammatoires et du monoxyde d'azote (NO). En cas de syndrome métabolique, les facteurs inflammatoires présents dans le sang portal induisent un défaut d'autophagie dans les LSEC, responsable d'une apoptose et d'une inflammation endothéliales, et favorisant la transition endothélio-mésenchymateuse et l'angiogenèse. Les LSEC altérées libèrent des cytokines inflammatoires (CCL2, CCL5, IL-6) et du TGF- $\beta$ (transforming growth factor- $\beta$ ). Ces altérations endothéliales contribuent au développement de l'apoptose hépatocytaire et de l'inflammation du tissu hépatique, favorisant ainsi le développement de la NASH et de la fibrose hépatique.

C motif chemokine ligand]2, CCL5, IL-1, IL-6, VCAM-1 [vascular cell adhesion molecule 1]) et libéraient d'importantes quantités de cytokines pro-inflammatoires (CCL2, CCL5, IL-6) par rapport à des LSEC témoins. Le défaut d'autophagie dans les LSEC induisait également une surexpression de gènes codant des marqueurs de transition endothéliomésenchymateuse ( $\alpha$-SMA [ $\alpha$-smooth muscle actin], TGF- $\beta 1$ [transforming growth factor-beta 1], collagène-1 $\alpha 2$ ) et d'angiogenèse, ainsi qu'une apoptose endothéliale (surexpression de la caspase- 3 activée et augmentation du marquage fluorescent d'apoptose en cytométrie en flux) [7]. Ces résultats indiquent que le défaut d'autophagie des LSEC modifie considérablement leur phénotype en induisant de l'inflammation et de l'apoptose endothéliale, et en favorisant l'angiogenèse et la transition endothélio-mésenchymateuse.

Afin d'évaluer l'effet du défaut d'autophagie dans les LSEC sur le développement de la NASH, nous avons généré des souris génétiquement déficientes pour l'autophagie spécifiquement dans leurs cellules endothéliales, et nous avons soumis ces souris à un régime riche en matières grasses pour induire le développement de la maladie. Nous avons observé des nodules à la surface du foie chez près de $80 \%$ des souris dont les cellules endothéliales avaient une autophagie déficiente et qui étaient soumises à ce régime, tandis que moins de $20 \%$ des souris témoins soumises au même régime alimentaire présentaient cet aspect du foie. Comparativement à ces souris témoins, les souris dont les cellules endothéliales avaient une auto- phagie déficiente avaient plus de lésions hépatiques (augmentation du taux plasmatique de la transaminase aspartate aminotransférase [ASAT], et de l'expression hépatique de la caspase-3) et une augmentation de l'inflammation hépatique (surexpression hépatique des gènes codant $C C L 2, C C L 5, C D 68^{1}$, et de la protéine V(AM-1). De plus, ces souris développaient une fibrose hépatique péri-sinusoïdale (surexpression hépatique des gènes codant TGF- $\beta 1$ et le collagène $1 \alpha 2$; surexpression de la protéine $\alpha$-SMA; extension de la fibrose, évaluée en histologie par coloration des fibres de collagène), tandis que les souris témoins n'en développaient pas [7]. Ces résultats montrent que le défaut d'autophagie dans les LSEC favorise les

\footnotetext{
${ }^{1}$ Une protéine lysosomale.
} 
lésions hépatocytaires et l'inflammation hépatique, et induit la fibrogenèse aux stades initiaux de la NASH.

Enfin, afin d'analyser le rôle de l'autophagie dans les LSEC aux stades avancés des maladies chroniques du foie, caractérisés par la présence d'une importante fibrose hépatique, nous avons traité des souris de génotype sauvage et les souris génétiquement déficientes pour l'autophagie dans les cellules endothéliales avec une molécule hépatotoxique, le tétrachlorure de carbone $\left(\mathrm{CCl}_{4}\right)$, pendant 6 semaines [8]. Dans ce modèle murin de fibrose hépatique, nous avons observé que la déficience en autophagie dans les cellules endothéliales favorisait le développement de la fibrose hépatique (surexpression hépatique des gènes codant TGF- $\beta 1$, collagène $1 \alpha 2$, et $\alpha$-SMA, extension de la fibrose évaluée en histologie par coloration des fibres de collagène) [7]. Ce résultat montre que le défaut d'autophagie dans les LSEC favorise le développement de la fibrose hépatique également à un stade avancé des maladies chroniques du foie.

En résumé, nous avons montré qu'une déficience de l'autophagie des LSEC est impliquée dans le développement de la NASH et des maladies chroniques du foie. Dans des conditions physiologiques, l'autophagie est active dans les LSEC et contribue à l'homéostasie hépatique, tandis que chez certains patients atteints de syndrome métabolique, l'autophagie des LSEC est altérée par les facteurs inflammatoires présents dans le sang portal. Ce défaut d'autophagie dans les LSEC induit une inflammation et une apoptose des cellules endothéliales, contribuant à l'inflammation et à la fibrose hépatiques, et favorisant le développement de la NASH (Figure 1). Plus généralement, nous avons montré pour la première fois que l'autophagie dans les LSEC joue un rôle antiinflammatoire et anti-fibrogénique, au même titre que l'autophagie dans les hépatocytes et les cellules de Kupffer. Nous suggérons que, parmi les patients atteints d'une stéatose hépatique, ceux pour lesquels l'autophagie dans les LSEC est déficiente évoluent vers la NASH, tandis que chez ceux dont l'autophagie dans les LSEC est fonctionnelle, la stéatose ne se complique pas d'une NASH. Cibler spécifiquement l'autophagie des cellules endothéliales pourrait donc constituer une approche thérapeutique intéressante pour prévenir la NASH ou pour la traiter. $\diamond$

Role of autophagy in liver sinusoidal endothelial cells in nonalcoholic steatohepatitis

\section{LIENS D'INTÉRÊT}

Les auteurs déclarent n'avoir aucun lien d'intérêt concernant les données publiées dans cet article.

\section{RÉFÉRENCES}

1. Estes C, Razavi H, Loomba R, et al. Modeling the epidemic of nonalcoholic fatty liver disease demonstrates an exponential increase in burden of disease. Hepatology $2018 ; 67: 123-33$

2. Friedman SL, Neuschwander-Tetri BA, Rinella M, et al. Mechanisms of NAFLD development and therapeutic strategies. Nat Med 2018 ; 24 : 908-22.

3. Diehl AM, Day C. Cause, pathogenesis, and treatment of nonalcoholic steatohepatitis. N Engl J Med 2017 ; 377 : 2063-72.

4. Hammoutene A, Rautou PE. Role of liver sinusoidal endothelial cells in non-alcoholic fatty liver disease. J. Hepatol $2019 ; 70: 1278-91$.

5. Poisson J, Lemoinne S, Boulanger C, et al. Liver sinusoidal endothelial cells: Physiology and role in liver diseases. J Hepatol $2017 ; 66$ : 212-27.

6. Allaire M, Rautou PE, Codogno P, et al. Autophagy in liver diseases: time for translation? J Hepatol 2019; $70: 985-98$.

7. Hammoutene A, Biquard L, Lasselin J, et al. A defect in endothelial autophagy occurs in patients with nonalcoholic steatohepatitis and promotes inflammation and fibrosis. J Hepatol 2020 ; 72 : 528-38.

8. Kantari-Mimoun C, Castells M, Klose R, et al. Resolution of liver fibrosis requires myeloid celldriven sinusoidal angiogenesis. Hepatology 2015 ; 61 2042-55.

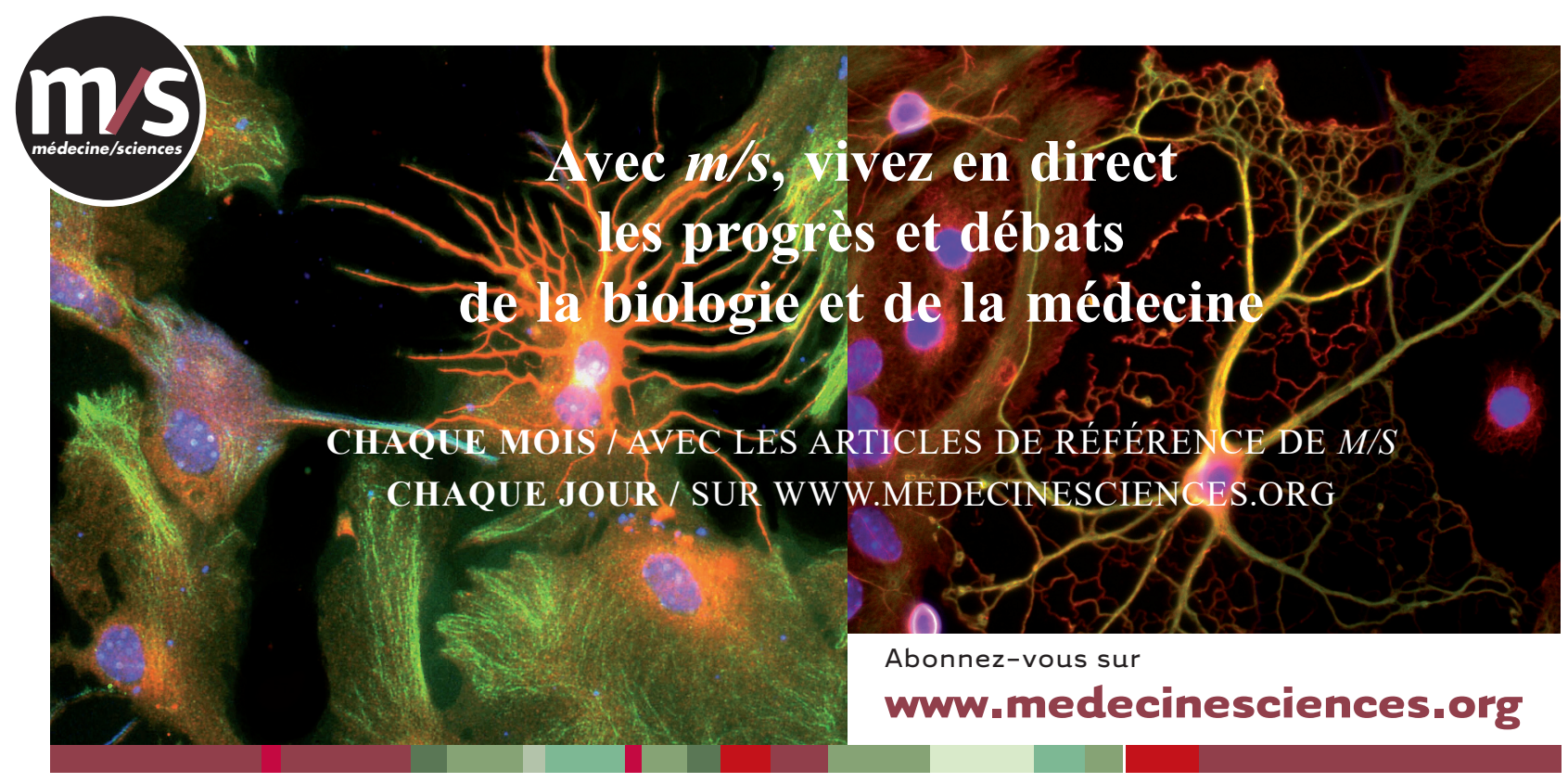

